I spoke of heat passing across a stratum of gas from one solid surface to another " as though there were, in contact with each solid surface, a layer of gas whose temperature is throughout the same as [it would perhaps have been been better to have said "determined by"] that of the contiguous solid."

I am fully aware of the ease with which one may be led into serious mistakes by trusting too implicity to such simplifying assumptions, and also that some of the particular suppositions made above would be inadmissible in a discussion of the general problem of the conduction of heat in gases; but I do not see any fallacy in employing them for the special purpose which I had in view in my last letter, namely, to show why I think that the flow of heat across a thin stratum of gas must be facilitated by diminishing the pressure of the gas. Prof. Osborne Reynolds's argument that "if there were a layer of uniform temperature, no beat would be transmitted," does not appear to me to be applicable to the case in question. It seems conceivable, as an extreme case, that, in a very thin layer of gas, between parallel solid surfaces maintained at different temperatures, the molecular movements might take place exclusively in the direction of the pernendicular to the bounding surfaces. In such a case the particles would move from side to side of the layer of gas with a uniform velocity, though the velocity one way would be greater than the velocity the other way, and heat would be transmitted across a layer of gas having the same temperature throughout. Such a condition, whether practically realisable or not, would, if I understand him aright, be the limiting case in one direction of what Mr. Stoney has called for shortness a "Crookes's layer:" the limiting case in the other direction being the ordinary condition of a gas, where the average velocity of the molecules is independent of direction. I venture to think that, in pointing out the results which must follow from the existence of a predominating direction of molecular motion, $\mathrm{Mr}$. Stoney has made a very important contribution to the kinetic theory of gases; and I do not see that his conclusions are in any way invalidated by its being shown that they are not in harmony with " the generally-accepted laws of gases," inasmuch as these laws are deduced from suppositions which expressly exclude the conditions he has investigated.

December 17

AlLow me to say a few words on what I believe to be the correct theory of the radiometer. This theory was given to me by Prof. Osborne Reynolds during spring of 1875 , and $I$ have found it capable of explaining every experiment on the subject with which I am acquainted.

The conservation of momentum is one of the laws of nature which even molecules do not break, and that law puts some restraints on the wonderful things which the shocks of molecules can accomplish. Imagine a vessel full of gas at a certain temperature. The centre of gravity of the gas and that of the vessel are supposed to be at relative rest. Suppose now that I increase the velocity of a certain number of molecules in a given direction, the centre of gravity of the gas will move relatively to the centre of gravity of the vessel, and no number of encounters between the molecules cau alter that motion until the momentum has been taken up by the vessel. If in any gas we have a passage of heat in a certain direction, we shall have a propagation of momentum owing to the fact that the molecules move more quickly in one direction than in the opposite one, and no number of encounters can alter that propagation. Where the momentum enters the gas and where it leaves it we observe certain forces. This is Prof. Reynolds's theory of the radiometer. It has been objected that an increased pressure on the cool side of the vanes of a radiometer will counterbalance the force acting on the blackened sides, when the dimensions of the vessel are large compared with the mean path of a molecule, but I do not think that such is the case. The following special case may make this point a little clearer. If the forces on the vanes are counterbalanced, the forces on the vessel must be counterbalanced as well. In the case of an ordinary radiometer the forces reduce to a couple, and I do not see how any crowding of molecules in one part of the vessel more than in another can produce a couple on the vessel. The whole problem is one of conduction of heat. All the experiments made by Mr. Crookes on cups, inclined vanes, \&c., admit of the same easy explanation as the fact that when a long and a short wire are connected with the poles of a battery, the current in the shorter wire will be the strongest. In a radiometer with inclined vanes, for instance, the temperature is the same on both sides, but the gradient of temperature is much larger on one side, and hence more heat will escape on that side. The dimensions of the vessel also have to be taken into account in the same way as the length of a wire bas to be taken into account when the strength of an electric current flowing through it has to be calculated. It is difficult to say exactly what takes place within very small distances from the hot surface, but it seems clear that any phenomenon, such as Prof. Carey Foster supposes to exist, must affect the passage of heat in the same way as the force on the vanes. As the careful researches of Messrs. Kundt and Warburg have shown that under great exhaustion the conduction of heat decreases and does not increase, I do not see how an increase in the force can take place.

The scientific world will judge how far Prof. Stoney has succeeded in establishing any new laws on the conduction of heat through gases. In justice, however, to Messrs. Provostaye and Dessains, whose experiments he calls to his aid, I wish to point out that their numerous experiments, with two exceptions, are in entire accordance with existing theories. At the time these experiments were made, no distinction was drawn between convection and true conduction. In order to deduce, therefore, the loss of heat due to true conduction, Prof. Stoney is obliged to subtract the effect due to convection currents. He draws, therefore, a curve representing the loss of heat due to this cause. All his conclusions must stand or fall with this curve, and I am afraid they must fall.

After Professors Clausius and Maxwell had deduced theoretically the coefficient of conductivity for gases, a series of celebrated experiments were made by Stefan, by Narr, by Plank, by Winkelmann, and last, but not least, by Kundt and Warburg. The influence of convection currents has been fully discussed in these papers and eliminated, and the conclusions arrived at by all these experimenters are fully in accordance with each other and with theory. It appears, as was expected, that when the effects of convection currents are eliminated, the coefficient of condur. tivity is independent of pressure until the dimensions of the vessel are comparable with the mean free path of a molecule, and that then the conductivity rapidly diminishes. It also appears that at the pressures at which Messrs. Provostaye and Dessains found that the loss of heat was independent of pressure, convection currents must have ceased to be appreciable, and therefore the great mass of their experiments is fully in agreement with later researches.

The only exception is found in the case of carbonic acid and nitrous oxide. These abnormal results were not confirmed by Messrs. Kundt and Warbirg in the case of carbonic acid, the only one of the two gases which they examined. Whoever reads their account of the difficulty they had in excluding the last traces of moisture, and considers the increased conductivity which such an admixture would! produce as the pressure diminishes, will have no difficulty in explaining the anomaly. At any rate I do not think Prof. Stoney will be inclined to base important conclusions on unconfirmed experiments on two gases in which we should expect the effect, owing to their density, to be particularly small. The discovery of Master Gerald Stoney, who found that a red hot wire was cooled when a tin can containing water was brought sufficiently close might, I think, have been foretold by the recognised theory. Prof. Stoney, no doubt, will find on reading over the literature on the subject, that what he calls penetration of heat, has hitherto been known under the name of conduction of heat, that it takes place at all pressures, and begins to disappear at the exact point at which he makes it appear.

The timely calculation of Mr.S. T. Preston in the August number of the Phil. Mag., shows that any theory of the radiometer which makes the action depend on the comparatively large ratio of the mean free path to the dimensions of the vessel, must necessarily be wrong.

Arthur Schuster

\section{The Proposed Channel Islands' Zoological Station, Aquarium, and Piscicultural Institute}

I AM very anxious that this project $x$ should succeed, mainly because of the facilities it will afford to inland aquaria, in procuring living animals cheaper, better, more variedly, and more systematically, than at present. This, I believe, will form the most profitable part of the undertaking.

\footnotetext{
I Referred to in NatURE, rol. xvii. F. Iө2,
} 